\title{
High prevalence of sleep and mood disorders in dry eye patients: survey of $\mathrm{I}, 000$ eye clinic visitors
}

This article was published in the following Dove Press journal:

Neuropsychiatric Disease and Treatment

31 March 2015

Number of times this article has been viewed

\section{Masahiko Ayaki \\ Motoko Kawashima \\ Kazuno Negishi \\ Kazuo Tsubota}

Department of Ophthalmology, Keio University School of Medicine,

Tokyo, Japan
Correspondence: Masahiko Ayaki

Department of Ophthalmology, Keio

University, 35 Shinanomachi, Shinjuku,

I608582 Tokyo, Japan

$\mathrm{Tel}+8 \mid 33353$ |2 I I

Fax +8I 333598303

Email mayaki@olive.ocn.ne.jp
Purpose: We aimed to explore the prevalence of probable sleep and mood disorders in eye clinic visitors.

Methods: This was a cross-sectional study. The participants were outpatients at six eye clinics from January through March, 2014. Outpatients were invited to complete a questionnaire containing the Pittsburgh Sleep Quality Index (PSQI) and Hospital Anxiety and Depression Scale (HADS). A final diagnosis was made, and patients were classified into six diagnostic groups. The main outcome measures were the scores of the PSQI and HADS among the diagnostic groups.

Results: A total 1,000 outpatients participated, and 730 patients (mean age $59.5 \pm 19.0$ years; 487 females) were analyzed after exclusion of children and patients diagnosed with healthy eyes, acute injury, or unilateral pseudophakia. The mean PSQI and HADS scores across all patients were 5.3 \pm 3.1 and $9.2 \pm 6.2$, respectively. For the diagnostic groups, the mean PSQI and HADS scores, respectively, were $5.7 \pm 3.3$ and $10.2 \pm 6.0$ for dry eye $(n=247), 5.4 \pm 3.2$ and $9.2 \pm 5.7$ for bilateral cataracts $(n=159), 5.3 \pm 3.3$ and $8.0 \pm 5.3$ for bilateral pseudophakia $(n=99)$, and, $5.0 \pm 3.1$ and $9.8 \pm 6.6$ for glaucoma $(n=109)$. Overall, $37.3 \%$ of patients were poor sleepers (PSQI $\geq 6$ ), and $45.5 \%$ had possible mood disorders (HADS $\geq 10$ ). Stepwise regression analysis revealed that the PSQI and HADS scores were significantly correlated with both age $(P<0.05)$ and the presence of dry eye $(P<0.05)$.

Conclusion: The prevalence of sleep and mood disorders was significantly higher in patients with dry eye. The present results suggest consultation-liaison psychiatry services may be beneficial among eye disease patients.

Keywords: depression, anxiety, cataract, liaison psychiatry, glaucoma

\section{Introduction}

Sleep disorders are generally associated with psychological, respiratory, urinary, rheumatic, or circulatory diseases. However, some of the presenting symptoms of eye disease, such as circadian misalignment, pain, and fear, could also be associated with sleep and psychiatric problems. For instance, circadian misalignment may develop in patients with glaucoma or cataracts, possibly leading to sleep disorders. Cataract is a light-deprivation disease, filtering the blue light that sensitizes intrinsically photosensitive retinal ganglion cells (ipRGCs). ${ }^{1}$ Recent studies demonstrated that cataract patients with yellow nuclear opacities had sleep disorders that improved after surgery to replace the opacified crystalline lens with an intraocular lens (IOL). ${ }^{2-4}$ Light therapy itself is also recognized as an effective intervention for circadian rhythm disorder, cognitive disorders, and depression in older adults. ${ }^{5}$ Glaucoma has a fundamental etiology of programmed cell death of retinal ganglion cells (RGCs), and its subgroup ipRGC expresses a newly identified opsin (melanopsin) that modulates photosynchronization of the superior chiasmatic nucleus, the master clock in the body. ${ }^{6-9}$ 
Eye disorders with chronic pain or discomfort, such as dry eye, trichiasis/entropion, chronic conjunctivitis, blepharitis, and other inflammatory diseases of the lid, conjunctiva, or cornea, may also be associated with sleep and mood disorders, via the trigeminal pathway..$^{10}$ Retinal diseases and glaucoma cause the most fear of blindness in patients since they directly affect vision and are often characterized by asymptomatic onset and worsening that disturbs quality of life. ${ }^{11,12}$

The purpose of this study was to investigate the prevalence of psychiatric problems in ophthalmic patients, in order to eventually provide better medical care for these patients. Therefore, we carried out a questionnaire-based survey in eye clinics to explore the prevalence of probable sleep and mood disorders.

\section{Materials and methods}

Six eye clinics in various practices and locations participated in this study. Institutional Review Board/Ethics Committee approval was obtained in each medical facility. Visitors to the eye clinics were invited to fill out questionnaires, which included the Pittsburgh Sleep Quality Index (PSQI) ${ }^{13}$ and the Hospital Anxiety and Depression Scale (HADS). ${ }^{14}$ Each questionnaire was self-administered during their visit and performed from January through March, 2014. Young participants below the age of 10 years were assisted by parents or guardians. The score for each scale was calculated according to separate algorithms, and the scores were then subject to analysis. The cutoff points for possible sleep and mood disorders were 5/6 for the PSQI and 9/10 for HADS, respectively. These questionnaires have been widely used for hospital-based surveys ${ }^{3,4,15,16}$ and are easy to answer, even by eye clinic visitors, because they do not contain difficult questions concerning severe psychiatric disease (eg, suicide and hallucination).

All outpatients were examined by board-certified ophthalmologists and certified orthoptists, and their diagnosis was classified as retina, glaucoma, cataract, IOL, dry eye, lid/conjunctiva, or health check, according to the physician's examination and the patient's primary problem. Our diagnostic criteria included the following: for retina, any vitreoretinal disease or symptom (eg, diabetic retinopathy, retinal detachment, and epiretinal membrane); for glaucoma, glaucomatous visual field loss, optic nerve abnormality, or elevated intraocular pressure requiring topical medication; for cataract, bilateral crystalline lens opacity accounting for subjective visual disturbance or decreased visual function; for IOL, bilateral pseudophakia more than 6 months after the second eye surgery; for dry eye, subjective or objective symptoms of the ocular surface requiring eye drops (eg, corneal epithelitis, corneal erosion, and symptoms such as dryness, pain, burning, and irritation); for lid/conjunctiva, chronic inflammatory lid or conjunctival disease (eg, conjunctivitis, chalazion, and blepharitis); and for healthy eyes, patients diagnosed with no clinically significant eye disease, including eye health checks and patients for the prescription of eyeglasses or contact lenses. There were some patients with multiple eye diseases, and these patients were classified into each diagnostic group.

Where appropriate, data are given as the mean \pm standard deviation. The mean score and proportion of patients with high values for the HADS and PSQI scores in each diagnostic group were compared among diagnostic groups. To identify which prominent eye diseases were complicated with psychiatric problems (sleep and mood disorders) in eye clinic visitors, a regression analysis was carried out with PSQI and HADS scores as dependent variables, and demographic (sex and age) and diagnostic groups as independent variables. Since some patients had multiple diagnoses and overlapped in diagnostic groups, a regression analysis was performed for each diagnostic group, adjusted for age and sex, and the results were compared among diagnostic groups. We found both sleep and mood disorders dominated in patients with dry eye and calculated the proportion of patients complicated with dry eye in the other diagnostic groups, where the chi-square test was used for comparison between IOL and cataract, ie preoperative and postoperative patients. Visual acuity was converted into LogMAR. All analyses were performed using StatFlex (Atech, Osaka, Japan) and SPSS version 21 (SPSS Inc, Chicago, IL, USA), with $P<0.05$ considered significant.

The research project conformed to the provisions of the 1995 Declaration of Helsinki (as revised in Edinburgh 2000).

\section{Results}

A total of 1,000 eye clinic visitors participated in the study. After exclusion of patients not relevant to the present analysis, namely, children $<10$ years $(\mathrm{n}=16)$ and patients diagnosed with healthy eyes $(n=173)$, acute injury $(n=40)$, or unilateral pseudophakia $(\mathrm{n}=41), 730$ patients were analyzed. The distributions of diagnosis, mean age, sex, and bestcorrected visual acuity are given in Table 1. Of all patients, $37.3 \%$ of patients were poor sleepers (PSQI $\geq 6$ ), and $45.5 \%$ had possible mood disorders (HADS $\geq 10$ ). Among the diagnostic groups, PSQI was the worst (highest) in the dry eye 
Table I Patient demographics and visual acuity

\begin{tabular}{|c|c|c|c|c|c|c|c|}
\hline Diagnostic group & Total & Retina & Glaucoma & IOL & Cataract & Dry eye & Lid/conjunctiva \\
\hline Number of patients ${ }^{\dagger}$ & 730 & 120 & 109 & 99 & 159 & 247 & 289 \\
\hline$\%$ female & 66.7 & 47.5 & 56.0 & 63.8 & 61.0 & 82.2 & 71.7 \\
\hline Age (years) & $59.5 \pm 19.0$ & $61.8 \pm 13.3$ & $68.2 \pm 13.9$ & $74.2 \pm 10.1$ & $72.7 \pm 10.9$ & $61.4 \pm 17.3$ & $51.1 \pm 21.2$ \\
\hline Median & 64 & 64 & 70 & 77 & 74 & 65 & 54 \\
\hline Range & $10-96$ & $15-88$ & $27-95$ & $28-88$ & $42-96$ & $11-95$ & $10-96$ \\
\hline LogMAR of worse eye & $0.10 \pm 0.35$ & $0.33 \pm 0.68$ & $0.08 \pm 0.20$ & $0.12 \pm 0.28$ & $0.26 \pm 0.45$ & $0.05 \pm 0.26$ & $0.02 \pm 0.14$ \\
\hline LogMAR of better eye & $0.00 \pm 0.10$ & $0.00 \pm 0.14$ & $0.00 \pm 0.11$ & $0.02 \pm 0.11$ & $0.07 \pm 0.12$ & $-0.01 \pm 0.08$ & $-0.0 \mathrm{I} \pm 0.08$ \\
\hline
\end{tabular}

Notes: Data are mean \pm standard deviation unless otherwise indicated. †Some of the patients had multiple diagnoses and so the sum is greater than 730 . Abbreviation: IOL, intraocular lens.

group (Table 2 and Figure 1). The HADS score was also the worst (highest) in the dry eye group (Table 2 and Figure 2). Regression analysis demonstrated age $(P<0.05)$ and the presence of dry eye $(P<0.05)$ were significantly correlated with PSQI and HADS (Table 3). The presence of cataracts and IOLs were inversely correlated with the HADS score.

Analysis of the PSQI subscales revealed more details about the sleep disorder of patients with dry eye, IOL, and cataract (Table 4). Patients with dry eye had the highest or second-highest PSQI scores for sleep duration, subjective sleep, and daytime dysfunction, while PSQI scores for sleep latency, sleep efficacy, sleep disturbances, and sleep medication were highest in patients in the cataract and IOL groups. HADS scores for anxiety and depression were highest or second-highest in the dry eye group, while glaucoma patients had the highest HADS score for depression. The proportion of cases complicated with dry eye was highest in the IOL and glaucoma groups (Table 4). The proportion of patients with dry eye in the IOL group was significantly higher than that in the cataract group $(P<0.001$, chi-square test $)$.

\section{Discussion}

The present study ascertained the sleep and mood condition of general eye clinic visitors, and revealed that some ocular problems were significantly associated with sleep and/or mood status. The most remarkable finding was that patients with dry eye had the worst PSQI and HADS scores. Recent reports ${ }^{17-19}$ described that dry eye is closely associated with depression or a deteriorated quality of life, resulting in considerable loss of productivity in the active working population. One potential etiology may be chronic stress due to the dry sensation or irritation of the ocular surface. It is notable that the scores for PSQI and HADS in the lid/ conjunctiva group were not as high as those in the dry eye group despite both clinical entities having common subjective symptoms, such as irritation, pain, and discomfort. Trichiasis and entropion, classified in the lid/conjunctiva group, are typical diseases, presenting with chronic irritation and a foreign body sensation, but the patients with these conditions did not record high scores for HADS or PSQI (data not shown). Our speculation is that patients with lid and conjunctival diseases can recognize the cause and location of their symptoms, thus the treatment effect of procedures or medication is as expected. In contrast, dry eye patients may be frequently anxious about their eyes, since symptoms of dry eye are lifelong, with unknown origin, and can worsen anytime due to seasonal, environmental, or local/systemic conditions. As such, dry eye may cause a constant feeling of discomfort or distress that causes mood disorders, especially in aged patients, who are especially sensitive to negative feelings and feelings of helplessness. Blindness as an outcome of eye disease may cause affective disorders,

Table 2 Diagnostic group and psychiatric results

\begin{tabular}{lllll}
\hline Diagnostic group & PSQI score & PSQI $\geq \mathbf{6}(\%)$ & HADS score & HADS $\geq \mathbf{I 0}$ (\%) \\
\hline Retina & $5.1 \pm 3.3$ & 35.0 & $9.6 \pm 5.5$ & $43.2^{*}$ \\
Glaucoma & $5.0 \pm 3.1$ & 34.9 & $9.8 \pm 6.6^{*}$ & 38.8 \\
IOL & $5.3 \pm 3.3$ & 38.8 & $8.0 \pm 5.3$ & 28.4 \\
Cataract & $5.4 \pm 3.2^{*}$ & $39.6^{*}$ & $9.2 \pm 5.7$ & 36.9 \\
Dry eye & $5.7 \pm 3.3^{*}$ & $42.1^{*}$ & $10.2 \pm 6.0^{*}$ & $43.5^{*}$ \\
Lid/conjunctiva & $5.2 \pm 3.1$ & 33.0 & $9.0 \pm 6.0$ & 41.8 \\
All cases & $5.3 \pm 3.1$ & 37.3 & $9.2 \pm 6.2$ & 45.5 \\
\hline
\end{tabular}

Notes: Data are mean \pm standard deviation unless otherwise indicated. *Highest and second-highest values for each parameter. Abbreviations: HADS, Hospital Anxiety and Depression Scale; IOL, intraocular lens; PSQI, Pittsburgh Sleep Quality Index. 


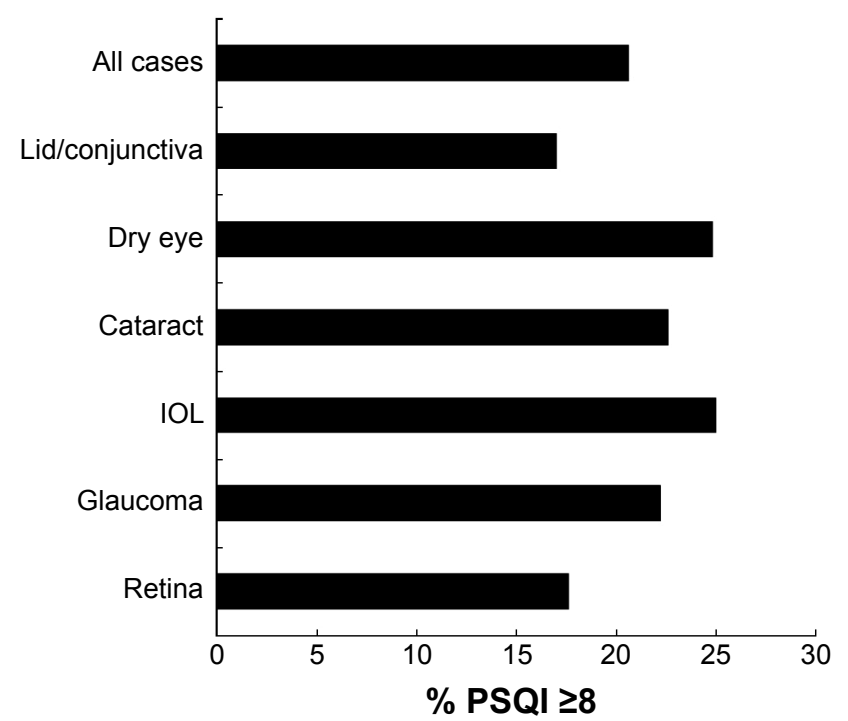

Figure I Proportion of patients with high values for the PSQI in each diagnostic group.

Notes: A score of PSQI $\geq 8$ indicates a clinically significant sleep disorder. The value for dry eye patients was the worst.

Abbreviations: IOL, intraocular lens; PSQI, Pittsburgh Sleep Quality Index.

while sleep disorders usually accompany mood disorders. This corresponds to the finding that both sleep and mood disorders dominated in patients with dry eye. However, further studies are warranted, as the cross-sectional study design and inclusion method of this survey did not allow the following questions to be answered: Is depression in these patients caused by severe dry eye? Is dry eye caused

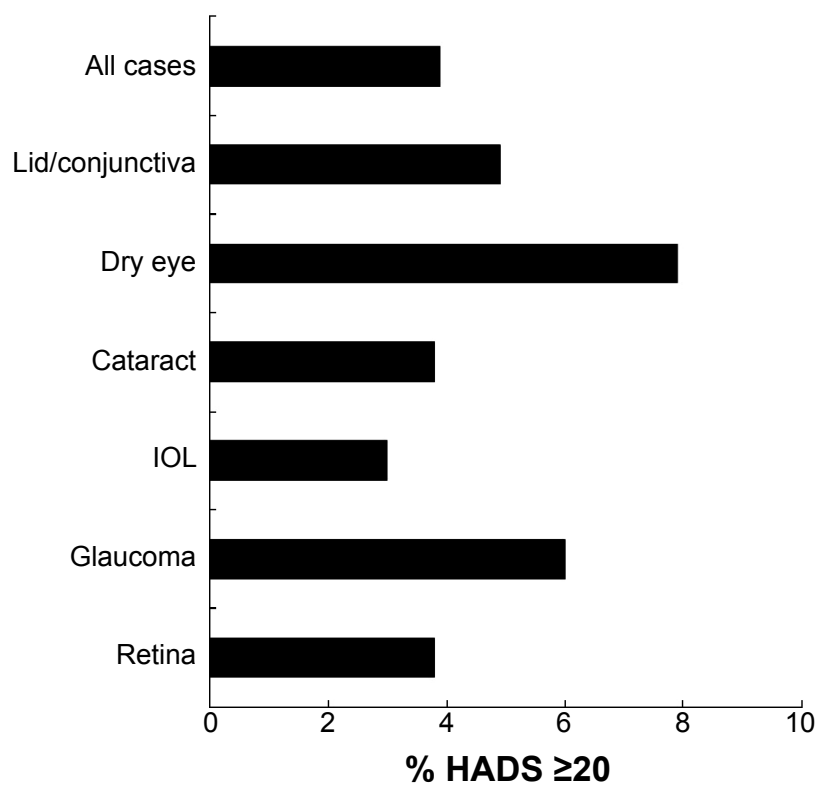

Figure 2 Proportion of patients with high values for the HADS in each diagnostic group.

Notes: A score of HADS $\geq 20$ indicates a clinically significant mood disorder. The value for dry eye patients was the worst.

Abbreviations: HADS, Hospital Anxiety and Depression Scale; IOL, intraocular lens.
Table 3 Regression analysis between diagnostic groups and parameters

\begin{tabular}{|c|c|c|c|c|}
\hline \multirow{2}{*}{$\begin{array}{l}\text { Diagnostic groups } \\
\text { and parameters }\end{array}$} & \multicolumn{2}{|l|}{ PSQI } & \multicolumn{2}{|c|}{ HADS } \\
\hline & $\beta$ & $P$-value & $\beta$ & $P$-value \\
\hline Retina & -0.01 & 0.75 & 0.01 & 0.86 \\
\hline Glaucoma & -0.04 & 0.35 & 0.02 & 0.51 \\
\hline IOL & -0.01 & 0.79 & -0.15 & $<0.001^{*}$ \\
\hline Cataract & 0.02 & 0.56 & -0.05 & $0.01 *$ \\
\hline Dry eye & 0.08 & $0.03^{*}$ & 0.08 & $0.03 *$ \\
\hline Lid/conjunctiva & -0.01 & 0.88 & -0.05 & 0.18 \\
\hline Age & 0.13 & $<0.00 I^{*}$ & 0.08 & $0.04 *$ \\
\hline Sex $x^{\dagger}$ & -0.03 & 0.33 & 0.04 & 0.27 \\
\hline
\end{tabular}

Notes: ${ }^{\dagger}$ Male $=I$, female $=0 . * P<0.05$, Pearson product moment correlation. Adjusted for age and sex.

Abbreviations: HADS, Hospital Anxiety and Depression Scale; IOL, intraocular lens; PSQI, Pittsburgh Sleep Quality Index.

by depression and/or sleeping disorders? Is dry eye in these individuals secondary to antidepressant medications?

Investigators have repeatedly reported significant improvement in the sleep quality of patients after cataract surgery $; 3,4$ however, surgical prognosis and the sustainability of sleep improvement after surgery were not determined in our study. The patients in the cataract group had minimal symptoms and mainly attended for a regular check-up, while the patients in the IOL group had their second eye surgery more than 6 months previously. Therefore, we could not evaluate the effect of surgery on sleep as in previous studies. However, Alexander et $\mathrm{al}^{3}$ reported the improvement in sleep after cataract surgery was not sustained for 12 months in either clear or yellow IOL cases. Espindle et $\mathrm{a}^{20}$ also described an improvement in the mental health score, using the 12-item Short Form Health Survey, but this was not sustained for 12 months. They speculated that the initial improvement might be transient due to a positive emotional state, better photo entrainment, and increased physical activity.

While there was a negative correlation of cataract and IOL with mood disorders, sleep disorders were prominent in patients with cataract and IOL, suggesting, in these groups at least, that sleep disorders are independent of mood disorders. Many older adults have comorbidities that affect sleep quality, including diabetes, brain disorders, cognitive disorders, and reduced endocrine function, as well as use of multiple medications. Their sleep disorder may recur after termination of a transient surgical effect. These patients had a stable condition and were robust enough to visit the eye clinic, compared with disabled older adults. The high prevalence (41\%) of dry eye in IOL patients may be associated with their sleep disorder as well as older age. Exacerbation 


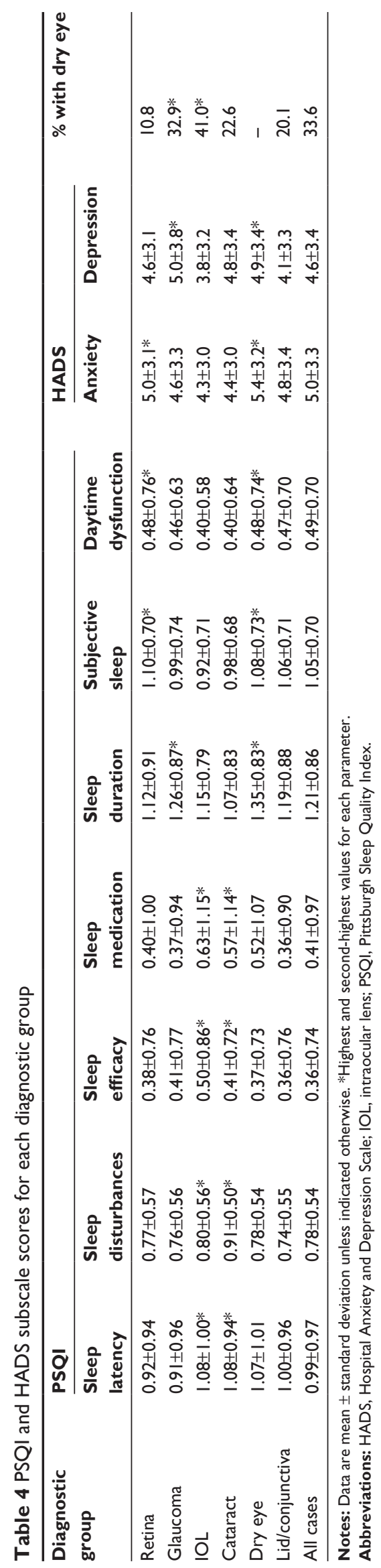

of dry eye after cataract surgery has been described..$^{21,22}$ A long surgery time, the surgical device used for aspirating irrigating solutions on the ocular surface, and insufficient lid hygiene have been suggested as risk factors for dry eye shortly after cataract surgery; ${ }^{21,22}$ however, further investigation will be necessary to explore whether cataract surgery and IOL itself have any association with long-term ocular surface problems.

Glaucoma patients did not have remarkably poor sleep despite their restricted visual field and presumably damaged ipRGCs. Their HADS score for depression was high, and some patients may be irritated by the disease and chronic use of medication. The high prevalence of dry eye in these patients may be due to topical medication containing preservatives. ${ }^{23,24}$ The sleep of glaucoma patients should be further evaluated by comparison with control patients with a normal visual field, adjusted for the presence of dry eye, especially taking into consideration that glaucoma patients do not have poor sleep, although their scores for mood disorders are high. Retina patients had mild anxiety, probably because many of their visits were for postoperative care.

This study had some limitations. Consecutive cases were enrolled to avoid bias, and the whole sample was heterogeneous with respect to age and sex. While the project was exploratory research, the results for each diagnostic group need further evaluation to clarify the relationship between the psychiatric disorders and the ocular pathologies. A control group was not included as each diagnostic group had a different age and sex distribution. There is a correlational nature of the findings in the present study, and hypotheses of causality would require evaluations at different time points. Further studies should be conducted to disclose psychiatric problems in eye disease patients.

In conclusion, the prevalence of sleep and mood disorders was highest in patients with dry eye, with some of these patients showing clinically significant scores for PSQI and HADS. Comprehensive management of eye clinic visitors with psychiatric problems, such as the use of consultationliaison psychiatry services, would contribute to better health care for these patients.

\section{Acknowledgments}

The authors thank Dr Yoshihiro Hashimoto, Dr Naoko Tachi, Dr Mariko Ishikawa, and Dr Naohisa Nezu for help with data collection, and Dr Takayuki Abe for help with statistical analysis. The authors acknowledge the assistance of InterBiotech (http://www.inter-biotech.com) with the English language editing of this manuscript. 
This study was presented at the Asia-ARVO 2015 conference, as a poster presentation with interim findings (abstract 513).

\section{Author contributions}

MA made substantial contributions to the conception and design of the study, collected and analyzed the data and wrote the manuscript draft. MK contributed to the design of the study, participated in interpreting the results and revised the paper for intellectual content. KN participated in interpreting the results and revised the paper for intellectual content. KT contributed to the design of the study, participated in interpreting the results and revised the paper for intellectual content. All authors read and approved the final version of the manuscript to be published. All authors agreed to be accountable for all aspects of the work in ensuring that questions related to the accuracy or integrity of any part of the work are appropriately investigated and resolved.

\section{Disclosure}

The authors report no conflicts of interest in this work.

\section{References}

1. Turner PL, Mainster MA. Circadian photoreception: ageing and the eye's important role in systemic health. Br J Ophthalmol. 2008;92(11): 1439-1444.

2. Kessel L, Siganos G, Jørgensen T, Larsen M. Sleep disturbances are related to decreased transmission of blue light to the retina caused by lens yellowing. Sleep. 2011;34(9):1215-1219.

3. Alexander I, Cuthbertson FM, Ratnarajan G, et al. Impact of cataract surgery on sleep in patients receiving either ultraviolet-blocking or blue-filtering intraocular lens implants. Invest Ophthalmol Vis Sci. 2014; 55(8):4999-5004.

4. Ayaki M, Muramatsu M, Negishi K, Tsubota K. Improvements in sleep quality and gait speed after cataract surgery. Rejuvenation Res. 2013; 16(1):35-42.

5. Stephenson KM, Schroder CM, Bertschy G, Bourgin P. Complex interaction of circadian and non-circadian effects of light on mood: shedding new light on an old story. Sleep Med Rev. 2012;16(5):445-454.

6. Quigley HA, Flower RW, Addicks EM, McLeod DS. The mechanism of optic nerve damage in experimental acute intraocular pressure elevation. Invest Ophthalmol Vis Sci. 1980;19(5):505-517.
7. Drouyer E, Dkhissi-Benyahya O, Chiquet C, et al. Glaucoma alters the circadian timing system. PLoS One. 2008;3(12):e3931.

8. Gracitelli CP, Duque-Chica GL, Moura AL, et al. A positive association between intrinsically photosensitive retinal ganglion cells and retinal nerve fiber layer thinning in glaucoma. Invest Ophthalmol Vis Sci. 2014; 55(12):7997-8005.

9. Hatori M, Panda S. The emerging roles of melanopsin in behavioral adaptation to light. Trends Mol Med. 2010;16(10):435-446.

10. Moulton EA, Becerra L, Borsook D. An fMRI case report of photophobia: activation of the trigeminal nociceptive pathway. Pain. 2009; 145(3):358-363.

11. Yuki K, Tanabe S, Kouyama K, et al. The association between visual field defect severity and fear of falling in primary open-angle glaucoma. Invest Ophthalmol Vis Sci. 2013;54(12):7739-7745.

12. Yokoi T, Moriyama M, Hayashi K, et al. Predictive factors for comorbid psychiatric disorders and their impact on vision-related quality of life in patients with high myopia. Int Ophthalmol. 2014;34(2):171-183.

13. Buysse DJ, Reynolds CF, Monk TH, Berman SR, Kupfer DJ. The Pittsburgh Sleep Quality Index: a new instrument for psychiatric practice and research. Psychiatry Res. 1989;28(2):193-213.

14. Zigmond AS, Snaith RP. The hospital anxiety and depression scale. Acta Psychiatr Scand. 1983;67(6):361-370.

15. Mabuchi F, Yoshimura K, Kashiwagi K, et al. Risk factors for anxiety and depression in patients with glaucoma. Br J Ophthalmol. 2012;96(6): 821-825.

16. Kong XM, Zhu WQ, Hong JX, Sun XH. Is glaucoma comprehension associated with psychological disturbance and vision-related quality of life for patients with glaucoma? A cross-sectional study. BMJ Open. 2014;4(5):e004632.

17. Uchino M, Schaumberg DA. Dry eye disease: impact on quality of life and vision. Curr Ophthalmol Rep. 2013;1(2):51-57.

18. Labbé A, Wang YX, Jie Y, Baudouin C, Jonas JB, Xu L. Dry eye disease, dry eye symptoms and depression: the Beijing Eye Study. Br J Ophthalmol. 2013;97(11):1399-1403.

19. Wen $\mathrm{W}, \mathrm{Wu} Y$, Chen $Y$, et al. Dry eye disease in patients with depressive and anxiety disorders in Shanghai. Cornea. 2012;31(6):686-692.

20. Espindle D, Crawford B, Maxwell A, et al. Quality-of-life improvements in cataract patients with bilateral blue light-filtering intraocular lenses: clinical trial. J Cataract Refract Surg. 2005;31(10):1952-1959.

21. Cho YK, Kim MS. Dry eye after cataract surgery and associated intraoperative risk factors. Korean J Ophthalmol. 2009;23(2):65-73.

22. Han KE, Yoon SC, Ahn JM, et al. Evaluation of dry eye and meibomian gland dysfunction after cataract surgery. Am J Ophthalmol. 2014; 157(6):1144-1150.e1.

23. Leung EW, Medeiros FA, Weinreb RN. Prevalence of ocular surface disease in glaucoma patients. J Glaucoma. 2008;17(5):350-355.

24. Iwasawa A, Ayaki M, Niwano Y. Cell viability score (CVS) as a good indicator of critical concentration of benzalkonium chloride for toxicity in cultured ocular surface cell lines. Regul Toxicol Pharmacol. 2013; 66(2):177-183.
Neuropsychiatric Disease and Treatment

\section{Publish your work in this journal}

Neuropsychiatric Disease and Treatment is an international, peerreviewed journal of clinical therapeutics and pharmacology focusing on concise rapid reporting of clinical or pre-clinical studies on a range of neuropsychiatric and neurological disorders. This journal is indexed on PubMed Central, the 'PsycINFO' database and CAS,

\section{Dovepress}

and is the official journal of The International Neuropsychiatric Association (INA). The manuscript management system is completely online and includes a very quick and fair peer-review system, which is all easy to use. Visit http://www.dovepress.com/testimonials.php to read real quotes from published authors. 